\title{
Comportement en flexion 3-points d'un matériau composite instrumenté par capteur à réseau de Bragg
}

\author{
Aurélie CORDELLE, Xavier CHAPELEAU, Monssef DRISSI- \\ HABTI $^{\mathrm{Q}}$
}

PRES LUNAM, IFSTTAR, Département Composants \& Systèmes (COSYS), CS4 44344 Bouguenais, FRANCE

a Pour toute correspondance : monssef.drissi-habti@ifsttar.fr

\begin{abstract}
RÉSUMÉ. Les matériaux composites sont de plus en plus utilisés comme matériaux de construction, notamment dans le génie civil. En revanche leur comportement en fatigue, et en particulier avec une sollicitation en flexion, est aujourd'hui encore mal connu. Ainsi il se développe des techniques de contrôle de santé pour surveiller les structures en continue, c'est le cas de la problématique du projet DECID2. L'intégration à cour de capteur à fibre optique dans un matériau obtenu par pultrusion permet de suivre la déformation de la structure tout au long de sa durée de vie. Cette étude s'intéresse à l'intégrité de l'instrumentation avec réseaux de Bragg non dénudés, notamment en termes de résistance statique et de caractère intrusif dans le matériau mais également en fatigue. L'application technologique sous-tendue consiste en le contrôle permanent de santé d'une structure de larges dimensions $(20 \mathrm{~m} \times 7 \mathrm{~m})$.

ABSTRACT.

Composite materials are increasingly used as building materials, especially in civil engineering. However their fatigue behavior, especially when submitted to bending stress is still poorly understood. Thus, the development of appropriate structural health monitoring techniques are highly needed and this is actually the aim of the National Project DECID2. The embedment of optical fiber sensors in a material obtained by pultrusion with the scope of monitoring the deformation of composite structures throughout its lifetime is one of the most promising way to get what is commonly called smart materials. This study focuses on the the evaluation of the integrity of the instrumentation with Fiber-Bragg-Gratings that is embedded in composites, particularly in terms of quasi-static loading as well as fatigue. The final goal is to validate this technique so that it can apply successfully in SHM of large dimensions structures $(20 \mathrm{~m} \times 7 \mathrm{~m})$.

MoTS-CLÉs: Flexion 3-points, Fatigue, Contrôle de santé structurale, Réseau de Bragg.

KEYWORDS: 3-point bending, fatigue, structural health monitoring, Fiber-Bragg-Gratings $(F B G)$
\end{abstract}

Revue. Volume $\mathrm{X}-\mathrm{n}^{\circ} \mathrm{x} /$ année, pages 1 à $\mathrm{X}$ 


\section{Extended Abstract}

Polymer-matrix composites are increasingly being used for the rehabilitation of structural components in civil engineering as well as for building sub-structures. Their good mechanical properties, excellent corrosion resistance, their relative stiffness / mass more elevated than steel, good processability and ease of installation are their main advantages. However, despite these, some key questions remain about their sustainability and environmental performance over the long term. Thus, the development of techniques for controlling structural health (Structural Health Monitoring, SHM) to monitor composite structures becomes mandatory.In this framework, the FUI Project (DGCIS Funds, French Ministry of Industry) Decid2) aims to build a demonstrator with large dimensions $(20 \mathrm{~m} \times 7 \mathrm{~m})$ entirely made with smart composites (composite instrumented by fiber optic sensors and ultrasonic patchs). The optical fiber used as a deformation sensor gives a diagnosis of structural health in real time. In addition, to avoid any degradation of the fiber or fiber bonding / structure due to the external environment, the project focuses on technology sensors inserted in the heart of the material. The platform is to be sought in fatigue and creep under low stress (well below the end of proportionality) outdoors, hence it is crucial to note that voluntarily Bragg gratings are not coated and mechanical tests are performed on specimens with a thickness comparable to the final profiles.In this study, the reliability of the instrumentation of the smart composite by embeddedsensors is checked during the manufacturing stage as well as during quasi-static and dynamic-fatigue tests under 3-point bending.

Several positive features have been shown about the insertion of optical fiber in the composite beams. Indeed, no optical fiber breaks during the pultrusion process was noted, a negligible intrusiveness, good signal-reliability was noted even when the composite specimens are submitted to high strain. However, a small error was noted below a strain of $0.1 \%$ and can be explained by the slight inability of the the optical fiber to reflect the real deformation in view of the thickening resulting from the operation of the photo-written FBG. A modification of the sheath could solve this problem but will make the fiber more brittle. It will be necessary to check the resistance of the new instrumentation in the new configuration. It must be remembered that the optical fiber is inserted for purposes of identifying abnormally high deformations which would be well above $0,1 \%$. Thus, it will be necessary to make a choice between greater precision of low-stress deformation or instrumentation that can sustain high strains. In this respect, given our target of embedding optical-fiber instrumentation into sections of several meters subject to fatigue or creep that can lead to the best FBG-measurement accuracy, leads us to favor a form of recoating thickness very similar to the original coating. 


\section{Introduction}

Les matériaux composites polymères sont de plus en plus utilisés pour la réhabilitation des systèmes de composants structuraux dans le génie civil et en tant que matériaux de constructions. Leurs bonnes propriétés mécaniques, leur excellente résistance à la corrosion, leur rapport rigidité/masse plus élevé que les aciers, leur bonne aptitude au traitement et leur facilité d'installation constituent leurs principaux avantages. Cependant, malgré ceux-ci, certaines questions essentielles demeurent quant à leur durabilité et leurs performances environnementales sur le long terme. Ainsi il se développe des techniques de contrôle de santé structurale: SHM (Structural Health Monitoring) afin de surveiller des structures en composite.

Dans ce cadre là, le projet FUI Decid2 (Projet Fonds Uniques Interministériels de la DGE, France) a pour ambition de construire un démonstrateur de $20 \mathrm{~m}$ x $7 \mathrm{~m}$ en matériau composite instrumenté par capteurs en fibre optique. La fibre optique utilisée comme capteur de déformation donne un diagnostic de santé structurale insitu et en temps réel. De plus, pour éviter toute dégradation de la fibre optique ou du collage fibre optique/structure due à l'environnement extérieur, le projet privilégie des technologies de capteurs insérés au cœur du matériau. La plateforme étant destinée à être sollicitée en fatigue et en fluage sous faibles contraintes (bien en dessous de la fin de proportionnalité) en extérieur, il est crucial de noter que volontairement les réseaux de Bragg ne sont pas revêtus et les essais mécaniques sont pratiqués sur des éprouvettes d'épaisseur comparable aux profilés finaux.

Dans cette étude, nous voulons vérifier la fiabilité de cette instrumentation à cœur : au niveau du procédé de fabrication, sur des essais statiques mais également en fatigue en flexion 3-points.

\section{Matériau et Essais}

\subsection{Matériau}

Le matériau considéré dans cette étude est un matériau composite à matrice du type vinylester et fibre de verre avec un très fort taux volumique $(\mathrm{Vf}=66 \%)$ qui lui confère une grandes rigidité et une résistance mécanique à la rupture élevée. Le vinylester est apprécié pour son faible coût de production, sa facilité de mise en œuvre mais particulièrement pour sa protection chimique des fibres contre la corrosion. Les fibres de verre quant à elles possèdent un bon rapport performances/prix.

Le procédé de fabrication retenu dans le cadre du projet Decid2, la pultrusion, permet d'obtenir des poutres à section constante sans limite de longueur. Ce procédé industriel est un atout majeur, surtout à l'heure actuelle où l'assemblage et le collage de matériaux composites constituent des barrières à son utilisation. Dans notre étude, la fibre optique est directement incorporée au sein du matériau pendant le 
procédé de pultrusion, au même titre que les fibres de renfort. On obtient ainsi un capteur intégré à cœur du matériau.

La section étudiée ici est rectangulaire et de dimensions $16 \mathrm{~mm}$ x $40 \mathrm{~mm}$. On peut noter que cette dernière est relativement importante comparée à la bibliographie. En effet dans une étude en fatigue où l'effet de taille est assez important, il est primordial de travailler avec des épaisseurs proches des poutres réelles, même si ces dernières n'ont pas la même géométrie.

\subsection{Flexion 3 points}

La passerelle prototype, ainsi que la plupart des éléments structuraux, sont sollicités en flexion. Ainsi pour se rapprocher au maximum des sollicitations réelles, les essais considérés sont des essais de flexion 3-points avec un écart entre les couteaux inférieurs de $260 \mathrm{~mm}$. Des premiers essais quasi-statiques (Figure 1) à déplacement de $1 \mathrm{~mm} / \mathrm{min}$ imposé ont permis de déterminer le module d'Young du matériau composite à $31 \mathrm{GPa}$. De plus, la fin de linéarité a pu être évaluée à environ $300 \mathrm{MPa}$, tout en gardant à l'esprit que pour un matériau avec un comportement très proche du comportement fragile, il est délicat d'établir rigoureusement cette valeur. Enfin, à l'aide d'essais d'émission acoustique, nous avons pu déterminer que les premiers endommagements se situaient pour des contraintes d'environ $130 \mathrm{MPa}$ (Fajoui et al., 2011).

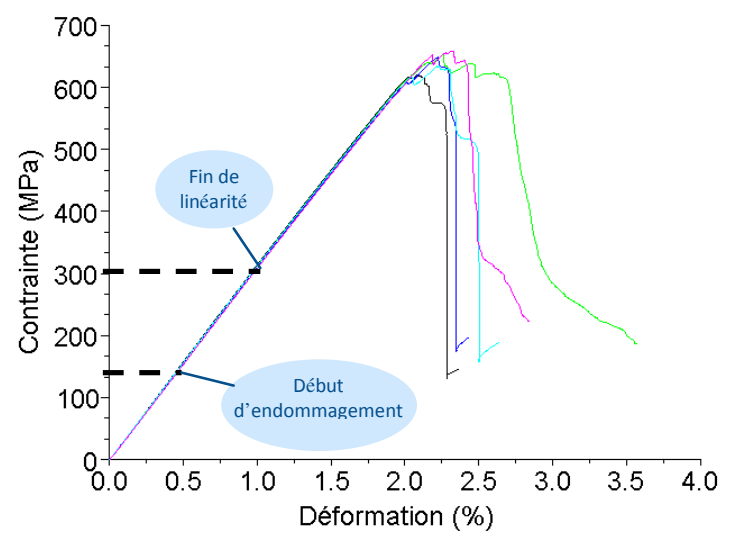

Figures 1. Courbe de flexion 3-points jusqu'à rupture sur 5 éprouvettes noninstrumentées. On repère sur ces courbes le début de l'endommagement et la fin de linéarité. 


\subsection{Essais de fatigue}

Les essais de fatigue en flexion 3-points de cette étude ont pour but de déterminer les évolutions des propriétés mécaniques pour des contraintes inférieures à la fin de proportionnalités. Ainsi les contraintes maximales étudiées se trouvent dans l'intervalle correspondant au début d'endommagement déterminé précédemment et la fin de linéarité. Le signal est sinusoïdal, avec une fréquence de 2 $\mathrm{Hz}$ sur 2 millions de cycles avec un rapport de charge de 0,1 . Des premiers essais non instrumentés nous permettent d'évaluer la perte de rigidité en fonction de la contrainte maximale imposée (Figure 2).

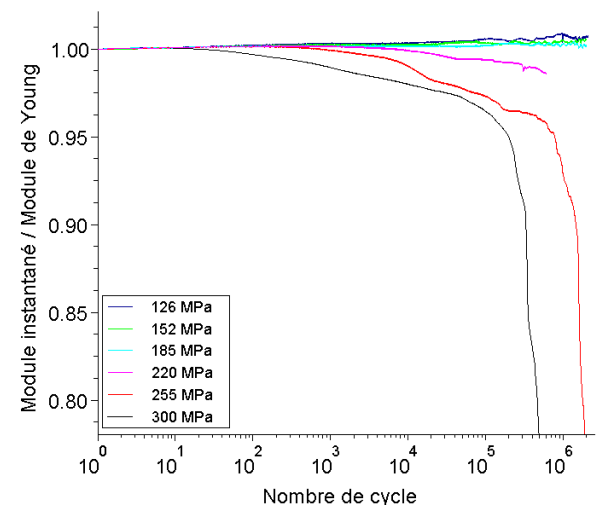

Figures 2. Baisse de rigidité des éprouvettes en fatigue à plusieurs niveaux de contrainte maximale.

\section{Instrumentation}

Les capteurs à fibre optique sont de plus en plus appréciés pour leurs nombreux avantages. Ce sont des capteurs multi-paramètres pouvant mesurer des températures, déformations, pression. Ils possèdent une grande stabilité de mesure et de faibles pertes. Ils sont interrogeables à distance et insensibles aux ondes électromagnétiques. De plus, leur souplesse leur permet d'instrumenter des structures « complexes». 


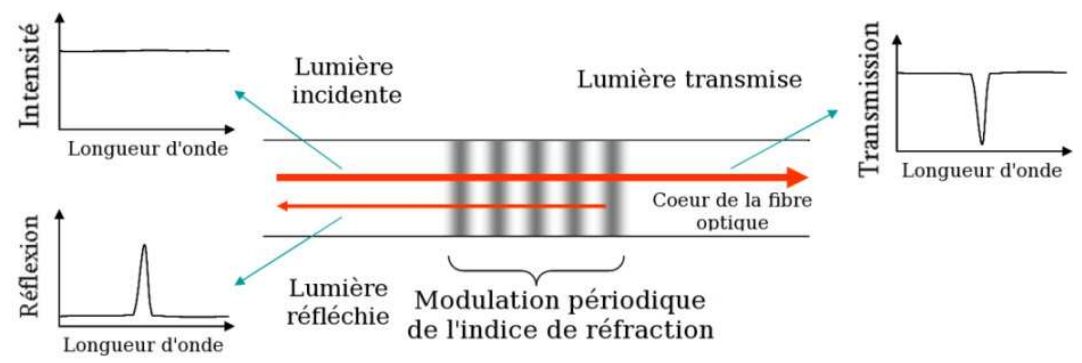

Figures 3. Principe de fonctionnement d'un capteur à réseau de Bragg.

Il existe deux types de capteurs, les capteurs répartis où la lecture de paramètres se fait tout au long de la fibre optique (Figure 3) et les capteurs distribués qui lit un paramètre ponctuellement sur la fibre optique. Les capteurs distribués, permettent une cadence de mesure très rapide, de l'ordre du kHz. C'est pourquoi ces capteurs sont privilégiés dans cette étude dynamique avec des capteurs à réseau de Bragg.

Un réseau de Bragg est photo-inscrit au cœur de la fibre optique avec un pas donné. A chaque pas correspond une longueur d'onde de Bragg (Figure 3), lorsqu'il y a dilatation thermique ou déformation de la fibre, le pas évolue, ainsi que la longueur d'onde de Bragg. C'est en étudiant le décalage des longueurs d'onde que l'on peut remonter aux déformations (Figure 4). Il est également possible de multiplexer une fibre optique avec plusieurs réseaux de Bragg dont les longueurs d'ondes de Bragg sont suffisamment dissociées.

$$
\lambda_{B}=2 . n_{\text {eff }} . \Lambda
$$

Avec $\lambda_{B}$ la longueur d'onde de Bragg, $\mathrm{n}_{\text {eff }}$ l'indice de réfraction effectif de la fibre optique et $\Lambda$ le pas du réseau.

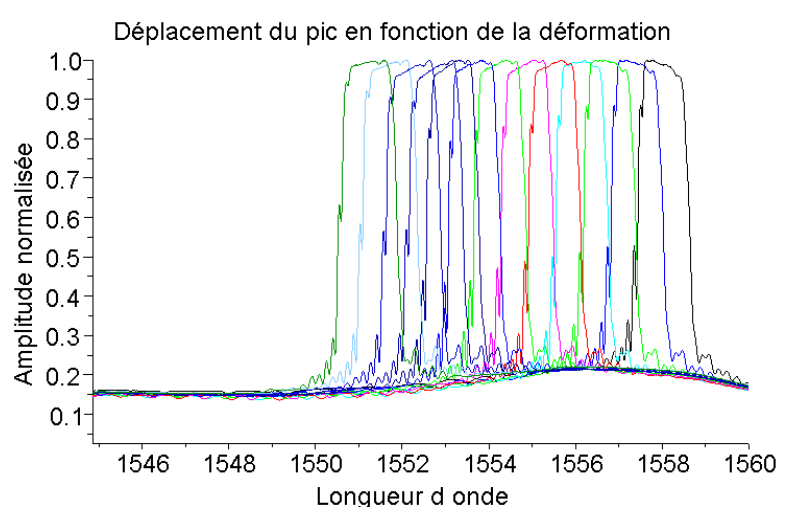


Figures 4. Déplacement du pic de la longueur d'onde de Bragg lors de l'augmentation de la déformation de la fibre optique, à température constante.

\section{Résultats et discussions}

\subsection{Résistance et caractère intrusif de la fibre optique}

Des essais préliminaires quasi-statiques ont été mis en oeuvre avec deux diamètres différents de fibre optique. Pour un diamètre de $125 \mu \mathrm{m}$, on a pu remarquer une rupture de la fibre optique avant rupture de l'éprouvette sur les trois essais, respectivement à 250,500 et $600 \mathrm{MPa}$, sachant que la rupture d'une éprouvette se situe entre 630 et $650 \mathrm{MPa}$. En revanche, sur tous les essais quasistatiques sur une éprouvette instrumentée avec une fibre optique de $80 \mu \mathrm{m}$, cette dernière reste saine jusqu'à la rupture de l'éprouvette. Par la suite, les choix technologiques se sont donc arrêtés sur le diamètre le plus faible qui possède une meilleure résistance mécanique au sein du matériau composite. En effet, plus celleci est faible, moins elle se comporte comme un défaut et elle se rapproche du comportement d'une fibre de renfort (environ $20 \mu \mathrm{m}$ ). Lors des essais de fatigue, sur la fibre optique de $80 \mu \mathrm{m}$, aucune rupture ou déchaussement avec la matrice n'a été occasionné.

De plus, également à ce diamètre, par rapport à une éprouvette non instrumentée, on observe une stabilité des propriétés mécaniques de l'éprouvette ainsi que la même durabilité sur des essais de fatigue. Cette observation semble cohérente car la fibre optique est constituée de verre, à l'instar des fibres de renfort. Il n'y a donc pas d'aberration en terme de différence de rigidité.

\subsection{Comportement du capteur et fiabilité du signal}

Outre les caractéristiques mécaniques, il est également essentiel de vérifier le comportement du capteur tout au long du cycle de vie d'une éprouvette, et donc d'une structure. Ainsi, la déformation lue par le réseau de Bragg est comparée à la déformation maximale de l'éprouvette calculée à partir de la mesure de la flèche. La fibre optique étant à cœur (Figure 5), puisque l'essai est un essai de flexion, cela nous donne deux mesures de déformation différentes. Ainsi pour valider le comportement du capteur, il suffit de vérifier que le rapport des déformations est constant tout au long des essais. Il est également important de noter que les réseaux de Bragg sont revêtus (re-coating) photo-inscription, dans le souci de les protéger contre le glissement mécanique différentiel lors d'essais de fatigue et qui peut entrainer la rupture de la fibre optique. Ceci va entrainer des erreurs aux faibles contraintes appliquées, comme nous le constaterons par la suite.

Les Figures 6 présentent respectivement ce rapport pour des essais quasistatiques jusqu'à rupture et de charge-décharge. On observe aisément sur tous les 
essais quasi-statiques, qu'à partir d'une certaine déformation de l'échantillon, nous obtenons un rapport constant qui traduit d'une bonne réponse du capteur à réseau de Bragg. Mais cette conclusion ne s'étend pas aux faibles déformations de l'échantillon où l'on peut remarquer une faible cohérence qui traduit un « retard de lecture à faible contrainte ».

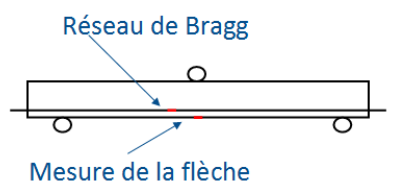

Figures 5. Position des déformations lues par les deux capteurs.

Plusieurs hypothèses ont été formulées pour expliquer ce « retard », nous avons pu, par le biais de nouveaux essais, écarter la plupart d'entre elles. Une première hypothèse consistait à mettre en cause la non-ponctualité du réseau de Bragg qui induirait des erreurs sur la lecture d'une déformation non uniforme pour un essai de flexion. Un essai de traction, où la fibre optique possède une déformation uniforme tout au long du réseau de Bragg, présente également ce « retard », cette hypothèse a donc été rejetée.
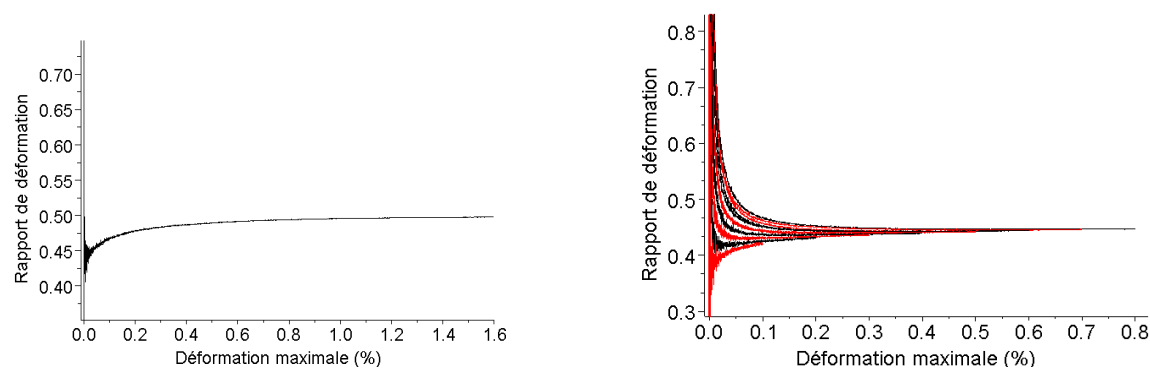

Figures 6. Rapport de la déformation lue par le capteur à réseau de Bragg par la déformation calculée à partir de la flèche maximale. Le premier graphique montre un essai quasi-statique jusqu'à rupture et le second un essai de charge décharge

L'erreur aurait également pu être due au déplacement de la traverse, l'utilisation d'un extensomètre sur l'essai de traction a également permis d'écarter cette seconde hypothèse. L'adhésion de la fibre optique au sein de la matrice aurait pu être mise en cause mais un essai avec une fibre collée sur l'échantillon (donc en changeant d'interface) montre toujours le même « retard ». Enfin, ce n'est également pas une 
mauvaise résolution du réseau de Bragg à faible déformation qui peut être mis en cause. En effet, pour des essais avec des réseaux situés à différent emplacements, le rapport peut varier du simple au quintuple et le «retard » se fait toujours à une contrainte donnée (environ $80 \mathrm{MPa}$ ) et non pas à une déformation du réseau de Bragg donnée.

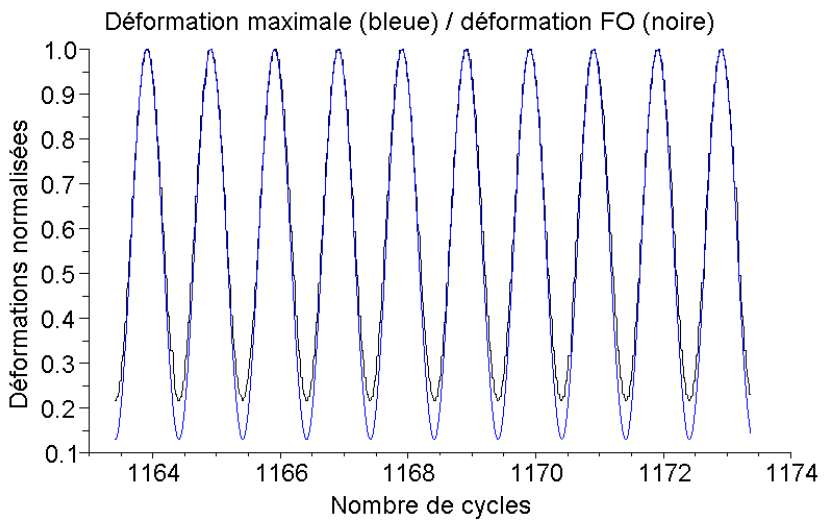

Figures 7. Déformations normalisées : calculée à partir de la flèche en bleue et lue par le capteur à réseau de Bragg en noir.

L'hypothèse retenue pour expliquer ce phénomène prend en compte un «effet tampon » de la gaine acrylate. En effet, afin d'inscrire une réseau de Bragg dans une fibre protégée par une gaine (l'acrylate dans notre cas), une mise à nu est effectuée dans la zone de photo-inscription. Une fois le réseau de Bragg inscrit, la fibre est reprotégée par de l'acrylate de façon manuelle. Ce qui introduit une surépaisseur locale. De ce fait, on peut expliquer l'aberration du réseau à faible valeurs de déformation simplement en considérant le frettage appliquée sur la fibre optique suite à la mise en flexion lors de l'essai. Ainsi, pour des valeurs de frettage inférieures à une valeur seuil, le réseau de Bragg reste insensible. Une fois cette valeur dépassée, la déformation est lue et donc fidèlement restituée. Afin de vérifier cette hypothèse, des essais complémentaires sont en cours en ce moment. Ils comportent des tests impliquant des fibres sans gaine et avec une gaine plus fine.

\section{Conclusion}

Cette étude démontre plusieurs points très positifs quant à l'insertion de fibre optique dans une poutre en matériau composite : pas de ruptures des fibres lors de la pultrusion, résistance de la fibre optique, caractère intrusif négligeable et une bonne fiabilité à forte déformation dans le matériau. 
A faible déformation en revanche, vraisemblablement, en dessous d'une déformation de $0,1 \%$, le frettage sur la fibre optique n'est pas suffisant compte tenu de la surépaisseur consécutive à l'opération de photo-inscription du réseau de Bragg. Une modification de la gaine pourrait pallier à ce problème mais rendrait la fibre optique plus fragile. Il sera nécessaire de vérifier la nouvelle résistance de l'instrumentation dans cette nouvelle configuration. Il faut rappeler que la fibre optique insérée a pour finalité de repérer des déformations anormalement élevées qui seraient bien supérieures à « limite de frettage ». Ainsi, il est nécessaire de faire un choix technologique entre une meilleure précision de la déformation à faible contrainte ou une résistance plus importante de l'instrumentation. A cet égard, compte tenu de notre souci de pérenniser la protection de la fibre optique insérée dans des profilés de plusieurs mètres soumis à la fatigue ou au fluage, tout en essayant d'obtenir la meilleure précision des mesures par réseaux de Bragg nous pousse à privilégier une forme de re-coating d'épaisseur très comparable au revêtement initial.

\section{Remerciements}

Ce travail a été conduit dans le cadre du projet FUI (fonds uniques interministériels de la DGE), Decid2. Monssef Drissi-Habti tient à remercier ces fonds, ainsi que la région Pays de la Loire pour le soutien financier.

\section{Bibliographie}

Chapeleau X., Drissi-Habti M., « Instrumentation à cœur des structures composites de courtes et moyennes portées en génie civil par des capteurs à fibre optique », Revue des Composites et des Matériaux Avancés, vol. 19, n 1, p. 7-24, 2009.

Chapeleau, X, Drissi-Habti, M and Tomonori, T, Embedded Optical Fiber Sensors in Civil Engineering Composite Structures, Materials Evaluation, Volume: 68 Issue: 4 Pages: 408-415, 2010.

Fajoui J., Terrien N., Dahmène F., Cordelle A., Chapeleau X., Drissi-Habti M., Suivi d'endommagement par émission acoustique dans un matériau composite à fibres de verre et à matrice vinylester obtenu par pultrusion et soumis à un chargement en flexion 3 points, à paraitre sur RCMA 